\title{
Influence of cannula positioning on brain injury during extracorporeal membrane oxygenation
}

\author{
Seong Cheol Jeong', Hee Jung Kim², Yeon Soo Shin ${ }^{3}$, Jung Wook Han ${ }^{1}$, Ju Yong Lim ${ }^{4}$, Ho Sung Son ${ }^{3,4}$ \\ ${ }^{1}$ Department of Thoracic and Cardiovascular Surgery, Uijeongbu St. Mary's Hospital, College of Medicine, The Catholic University of Korea, \\ Uijeongbu-si, Gyeonggi-do, Korea; ${ }^{2}$ Department of Thoracic and Cardiovascular Surgery, Asan Medical Center, College of Medicine, University of \\ Ulsan, Seoul, Korea; ${ }^{3}$ Korea Artificial Organ Center, Korea University, Seoul, Korea; ${ }^{4}$ Department of Thoracic and Cardiovascular Surgery, Korea \\ University Medical Center, Seoul, Korea \\ Contributions: (I) Conception and design: SC Jeong, HJ Kim, HS Son; (II) Administrative support: HS Son; (III) Provision of study materials or \\ patients: HS Son; (IV) Collection and assembly of data: YS Shin; (V) Data analysis and interpretation: SC Jeong, HJ Kim, YS Shin, JW Han, JY Lim; \\ (VI) Manuscript writing: All authors; (VII) Final approval of manuscript: All authors. \\ Correspondence to: Ho Sung Son. Department of Thoracic and Cardiovascular Surgery, Korea University Medical Center, Inchon-Ro 73, Sungbuk- \\ Gu, Seoul, Korea. Email: hssonmd@korea.ac.kr.
}

Background: In veno-arterial extracorporeal membrane oxygenation (V-A ECMO), a patient is cannulated using either an atrio-aortic technique (central type ECMO; cECMO) or a femoro-femoral technique (peripheral type ECMO; pECMO). The direction of the pump flow at the aortic arch is anterograde from the ascending aorta in $\mathrm{cECMO}$ and retrograde from the descending aorta in pECMO. Hemodynamic differences from the position of the cannulas may influence the brain differently. To evaluate the effect of ECMO cannula positioning on the brain, hemodynamic data and plasma biomarkers were collected.

Methods: Eight pigs were randomly divided into the cECMO group $(n=4)$ or pECMO group $(n=4)$. ECMO was administered for 6 hours at a pump flow rate based on the mean flow of the ascending aorta. Mean arterial pressure (MAP), mean arterial flow (MAF), energy equivalent pressure (EEP), and surplus hemodynamic energy (SHE) were measured in the brachiocephalic artery every 30 minutes. During ECMO treatment, plasma was collected for analysis of interleukin-6 (IL-6), S100B, glial fibrillary acidic protein (GFAP), and neuron-specific enolase. The data were analyzed using the Mann-Whitney $U$ tests, and repeated measures ANOVAs; significance was set at $\mathrm{P}<0.05$.

Results: MAP and EEP at 1 and at 3 hours, MAF at all measured times, and SHE at 1 hour and 6 hours were significantly higher in the pECMO group. There was no significant difference in the levels of brain injury biomarkers between cECMO and pECMO groups.

Conclusions: The hemodynamic data showed that pECMO was superior to cECMO. Based on the biomarker data, neither pECMO nor cECMO for 6 hours caused evidence of brain injury.

Keywords: Cannula; hemodynamics; extracorporeal membrane oxygenation (ECMO); brain injury

Submitted Jul 17, 2018. Accepted for publication Oct 15, 2018.

doi: $10.21037 /$ jtd.2018.10.74

View this article at: http://dx.doi.org/10.21037/jtd.2018.10.74

\section{Introduction}

Extracorporeal membrane oxygenation (ECMO) is used to treat acute respiratory syndrome and cardiogenic shock. ECMO drains the venous blood, removes carbon dioxide and oxygenates the blood through an artificial lung, and returns the blood to either the venous system ( $\mathrm{V}-\mathrm{V}$ ECMO, veno-venous) or the arterial system (V-A ECMO, venoarterial). When veno-arterial access is used for cardiac support in central ECMO (cECMO), the right atrium is cannulated to drain the venous blood, and the ascending aorta is cannulated to return the oxygenated blood. The 
femoral artery and vein are chosen for cannulation in peripheral ECMO (pECMO). In cECMO, blood from the left ventricular outflow joins returned blood flow from proximal ascending aorta in the same direction at the level of the ascending aorta. In pECMO, returned blood flow from the femoral artery joins the blood from the left ventricular outflow near the aortic arch in the opposite direction. These hemodynamic differences resulting from the cannula positioning may have varying influences on the brain.

Cerebral complications are a major risk factor for mortality during ECMO (1). The causes of cerebral complications include air embolism, thromboembolism, cerebral hemorrhage due to coagulopathy, and hemodynamic changes due to non-pulsatile flow (2) and also circulating blood exposure to foreign surface of ECMO circuit, blood flow shear stress, cytokine and complement system result in the abnormal activation of systemic immune response (3). The reported rate of cerebral complications is lower than expected, because CT and/or MRI evaluation for cerebral injury is limited during ECMO therapy $(4,5)$. For these reasons, other methods have been used for detecting brain injury. Seizure activity (6), abnormal electroencephalograms, and somatosensory evoked potentials (7) have all been used to evaluate cerebral injury. Neurological complications are also estimated by measuring various brain biomarkers. An elevated level of S100B has been associated with intracranial hemorrhage in infants treated with ECMO (8), and high glial fibrillary acidic protein (GFAP) during ECMO has been associated with acute brain injury and death (9).

Most previous studies using hemodynamic monitoring to determine the effects of ECMO on the brain did not take into consideration the cannula insertion site. The present study, however, compared the hemodynamic effects and plasma biomarkers for brain injury between pigs receiving cECMO and pECMO. To evaluate the hemodynamic effects on the brain according to the site of cannula insertion, we calculated the EEP and SHE by directly measuring blood flow and blood pressure in the brachiocephalic artery in both groups. The mean arterial pressure (MAP) calculates from measured systolic and diastolic blood pressure values. EEP is a marker that quantifies hemodynamic status in nonpulsatile and pulsatile ECMO by calculating blood flow and blood pressure in pulsatile flow, using waveforms. Pulsatility is defined as a pulse pressure greater than $15 \mathrm{mmHg}$, and pulsatile flow depends more on the difference in energy than the blood pressure (10). Pulsatile pressure-flow waveforms should be quantified in terms of hemodynamic energy levels because generation of pulsatile flow depends upon an energy gradient (11). The difference between EEP and MAP, i.e., SHE, is the "extra energy" that exists only if there is some degree of pulsatility in the pressure or flow. Under $100 \%$ non-pulsatile conditions, the SHE is zero (11). SHE acts as extra energy to more effectively maintain the patency of the capillaries and microcirculation. In this study, pulsatility was not eliminated, because ECMO in the clinical field was implemented without left ventricular fibrillation or clamping of the aorta. Therefore, in addition to MAP and MAF, EEP and SHE were selected as comparative hemodynamic markers.

This study also evaluated the effects of different cannula positions on brain injury during ECMO by comparing plasma levels of various brain biomarkers (S100B, GFAP, and NSE: neuron specific enolase) and the proinflammatory cytokine interleukin-6 (IL-6). These markers are secreted by brain tissue into the systemic circulation during brain damage and can increase in concentration following hemodynamic damage to the blood-brain barrier.

\section{Methods}

\section{Subjects}

This study was approved by the Guide for the Care and Use of Laboratory Animals issued by the Korea University School of Medicine (KUIACUC-2015-63). Eight female Yorkshire swine weighing between 33.8 and $50 \mathrm{~kg}$ were chosen for this study.

\section{Anesthesia and monitoring}

After intramuscular injection of Zoletil $(4.4 \mathrm{mg} / \mathrm{kg})$, a $21 \mathrm{G}$ venous catheter was placed into the ear vein, and the animal was intubated with a 6-7 Fr endotracheal tube. Mechanical ventilation was maintained with a $1: 1 \mathrm{~N}_{2} \mathrm{O} / \mathrm{O}_{2}$ gas mixture, tidal volume of $10-15 \mathrm{~mL} / \mathrm{kg}$, and a rate of $20-25$ breaths $/ \mathrm{min}$. ECG was monitored continuously, and core body temperature was monitored using a rectal probe.

\section{Surgical protocol}

After performing a median sternotomy, a $16 \mathrm{G}$ angiocatheter was placed into the innominate vein for intravenous fluid administration. A $16 \mathrm{~mm}$ perivascular flow probe (16 PAU, TS 420, TransonicSystems Inc., Ithaca, 
NY, USA) was placed near the ascending aorta. The average flow rate $(\mathrm{mL} / \mathrm{min})$ of the ascending aorta was assumed to be $100 \%$ of the animal's cardiac output. We used this average flow rate to set the mean ECMO flow rate in each pig. We investigated the difference between cECMO and pECMO in the same condition between the groups, so it was reasonable that we proceeded with sustaining ECMO flow based on ascending aorta flow. An $18 \mathrm{G}$ angiocatheter was inserted into the brachiocephalic artery to monitor the pressure and a $5-\mathrm{mm}$ perivascular flow probe (5 PSB, TS 420, TransonicSystems Inc., Ithaca, NY, USA) was placed near the brachiocephalic artery in order to measure flow rate. After heparinization $(3 \mathrm{mg} / \mathrm{kg}$ ), animals were randomly assigned to one of two groups: the cECMO group or the pECMO group.

Arterial cannulation of the ascending aorta (12 Fr DLP arterial cannula; Medtronic, Inc., Minneapolis, MN, USA) and venous cannulation of the right atrium through the left femoral vein (17 Fr DLP venous cannula; Medtronic, Inc., Minneapolis, MN, USA) were performed in the cECMO group. Arterial cannulation of the left femoral artery (14 Fr DLP femoral arterial cannula; Medtronic, Inc., Minneapolis, MN, USA) and venous cannulation of the right atrium though the left femoral vein (17 Fr DLP femoral venous cannula; Medtronic, Inc., Minneapolis, $\mathrm{MN}$, USA) were performed in the pECMO group. These cannulas were connected to a centrifugal pump (Quadrox PLS, Maquet, Germany), and ECMO was maintained for 6 hours. At the end of the experiment, the animals were sacrificed while anesthetized per the study protocol.

\section{Measurement of hemodynamic change}

Hemodynamic data [MAP; mean arterial flow (MAF); energy equivalent pressure (EEP); surplus hemodynamic energy (SHE)] were collected using the pressure transducer connected to the angiocatheter and a transit time flow meter (TS420; TransonicSystems Inc., Ithaca, NY) connected to the flow probe in the brachiocephalic artery. Data were analyzed using the LabVIEW software (National Instruments, Austin, TX, USA), which saved data at intervals of 1/1,000 second for 60 seconds before and after initiation of extracorporeal circulation and at 30-minute intervals during the 6-hour ECMO treatment period. We compared the data between the cECMO and pECMO groups from each hour time point.

EEP was defined by the following equation (12):

$\operatorname{EEP}(\mathrm{mmHg})=\left(\int \mathrm{fpdt} / \int \mathrm{fdt}\right)$
Where, $\mathrm{f}$ is the pump flow rate $(1 / \mathrm{min}), \mathrm{p}$ is the arterial pressure $(\mathrm{mmHg})$, and the time integrals are over one pulse cycle.

SHE was defined as follows (11):

SHE $\left(\mathrm{ergs} / \mathrm{cm}^{3}\right)=1,332 \times(\mathrm{EEP}-\mathrm{SHE})$

The constant $(\mathrm{n}=1,332)$ converts the pressure unit from millimeters of mercury to dynes per $\mathrm{cm}^{2}$.

\section{Measurement of Brain biomarkers (S100B, GFAP, NSE) and proinflammatory cytokine (IL-6)}

Blood samples from the innominate vein were collected before initiation of ECMO and at 1, 3 and 6 hours during ECMO. Blood was centrifuged for 15 minutes at 2,500 rpm at $4{ }^{\circ} \mathrm{C}$, and the samples were then stored at $-70^{\circ} \mathrm{C}$. Plasma levels of biomarkers from the samples were measured using an ELISA kit (Cusabio Biotech Co., Ltd., Wuhan, China) per the manufacturer's instructions.

\section{Statistical analysis}

Sample power data analysis in a Pilot study showed that a sample size of 4 in each group will be sufficient to detect a difference in mean hemodynamic data, assuming a standard deviation of 5 points, a minimum mean difference of $20 \mathrm{mmHg}$, a power of $80 \%$, and a significance level of $5 \%$.

Hemodynamic data and plasma levels of biomarkers are expressed as the median value (range, minimum-maximum). To determine the difference in the baseline characteristics and hemodynamic data between two groups, data were analyzed using the Mann-Whitney $U$ tests. To compare plasma levels of biomarkers over time within each group and differences between groups, a repeated measures analysis of variance was used. Significance was defined by $\mathrm{P}<0.05$. All statistical analysis was performed with the Statistical Package for the Social Sciences (SPSS) version 22.0 (IBM SPSS Statistics for Windows; IBM Corp, Armonk, NY, USA).

\section{Results}

\section{Baseline characteristics}

The median weight of the pigs was $41.0 \mathrm{~kg}$ (range, 35.6$46.0 \mathrm{~kg}$ ) in the cECMO group, and $42.0 \mathrm{~kg}$ (range, $34.3-$ $45.0 \mathrm{~kg}$ ) in the pECMO group; there was no significant difference in weight between the two groups. The flow through the ascending aorta before ECMO treatment 
Table 1 Characteristics of swine

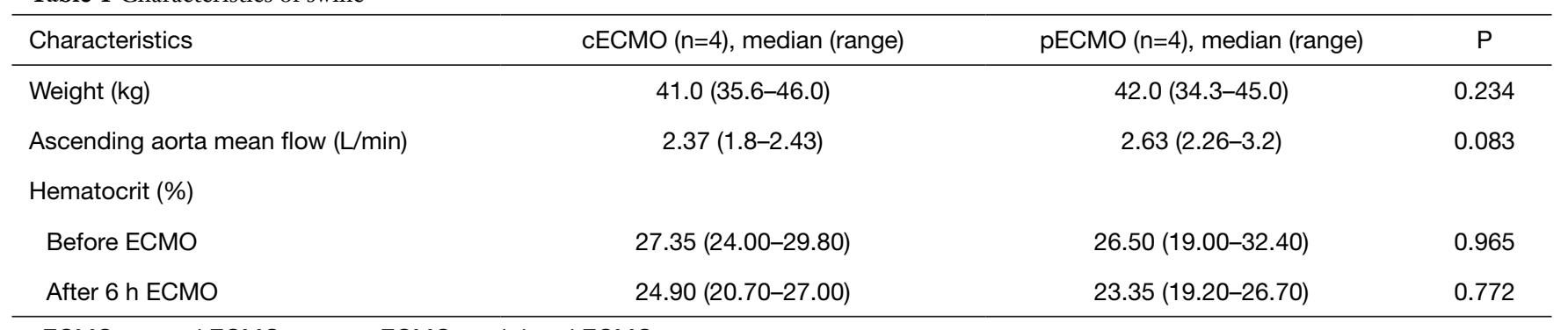

cECMO, central ECMO group; pECMO, peripheral ECMO group.

Table 2 Hemodynamic parameters prior to ECMO treatment

\begin{tabular}{|c|c|c|c|}
\hline Hemodynamic parameters & cECMO, median (range) & pECMO, median (range) & $\mathrm{P}$ \\
\hline MAF (L/min) & $0.58(0.44-0.70)$ & $0.80(0.41-1.02)$ & 0.248 \\
\hline EEP $(\mathrm{mmHg})$ & $61.43(54.60-73.63)$ & $56.20(51.04-61.31)$ & 0.386 \\
\hline SHE (ergs/cm³) & $2,118.32(1,651.42-5,119.95)$ & $5,261.74(2,257.30-16,237.14)$ & 0.149 \\
\hline
\end{tabular}

cECMO, central ECMO group; pECMO, peripheral ECMO group; MAP, mean arterial pressure; MAF, mean arterial flow; EEP, energy equivalent pressure; SHE, surplus hemodynamic energy.

used to set the ECMO flow rate was also not significantly different between the two groups (Table 1). All eight pigs survived the 6-hour ECMO treatment.

\section{Hemodynamic changes}

Increasing the volume of the plasma solution were used to support animal blood pressure without medication. Hemodynamic data were not significantly different between the two groups before ECMO treatment (Table 2). Both MAP and EEP were significantly higher in the pECMO group compared to the cECMO group at 1 and 3 hours. MAF was significantly higher in the pECMO group during the entire 6-hour treatment period. SHE was significantly higher at 1 and 6 hours in the pECMO group (Figure 1, Table 3).

\section{Brain biomarkers (S100B, GFAP, NSE) and proinflammatory cytokine (IL-6)}

There was no statistical difference in plasma levels of biomarkers (S100B, GFAP, NSE) or IL-6 between the cECMO and the pECMO group during the 6-hour treatment period, nor were any statistical changes observed over time in either group (Figure 2).

\section{Discussion}

Previous studies have reported the effects of ECMO on various organs, including the brain. Short et al. reported a decrease in the autoregulation of cerebral blood flow and cerebral oxygen consumption in the V-A ECMO group compared to the non-ECMO group in newborn lambs (13). Hunter et al. showed as much as a $25 \%$ decrease in cerebral blood flow with V-A ECMO compared to V-V ECMO by measuring temporal lobe perfusion with laser Doppler flowmetry (14). Liem et al. used near-infrared spectrophotometry and observed a decrease in cerebral oxygenation after ECMO in 24 neonates who underwent carotid artery ligation (15).

This study showed a significantly higher MAP and EEP at 1 and 3 hours, higher MAF during all 6 hours, and higher SHE at 1 and 6 hours in the pECMO group. These results could be due to the non-pulsatile reverse flow from pECMO meeting the pulsatile forward flow from the left ventricle near the aortic arch; this could result in greater flow through the brachiocephalic artery compared to cECMO. In contrast, with central ECMO, non-pulsatile flow meets the pulsatile forward flow from the left ventricle, which leads to greater flow through the descending aorta than through the brachiocephalic artery. 

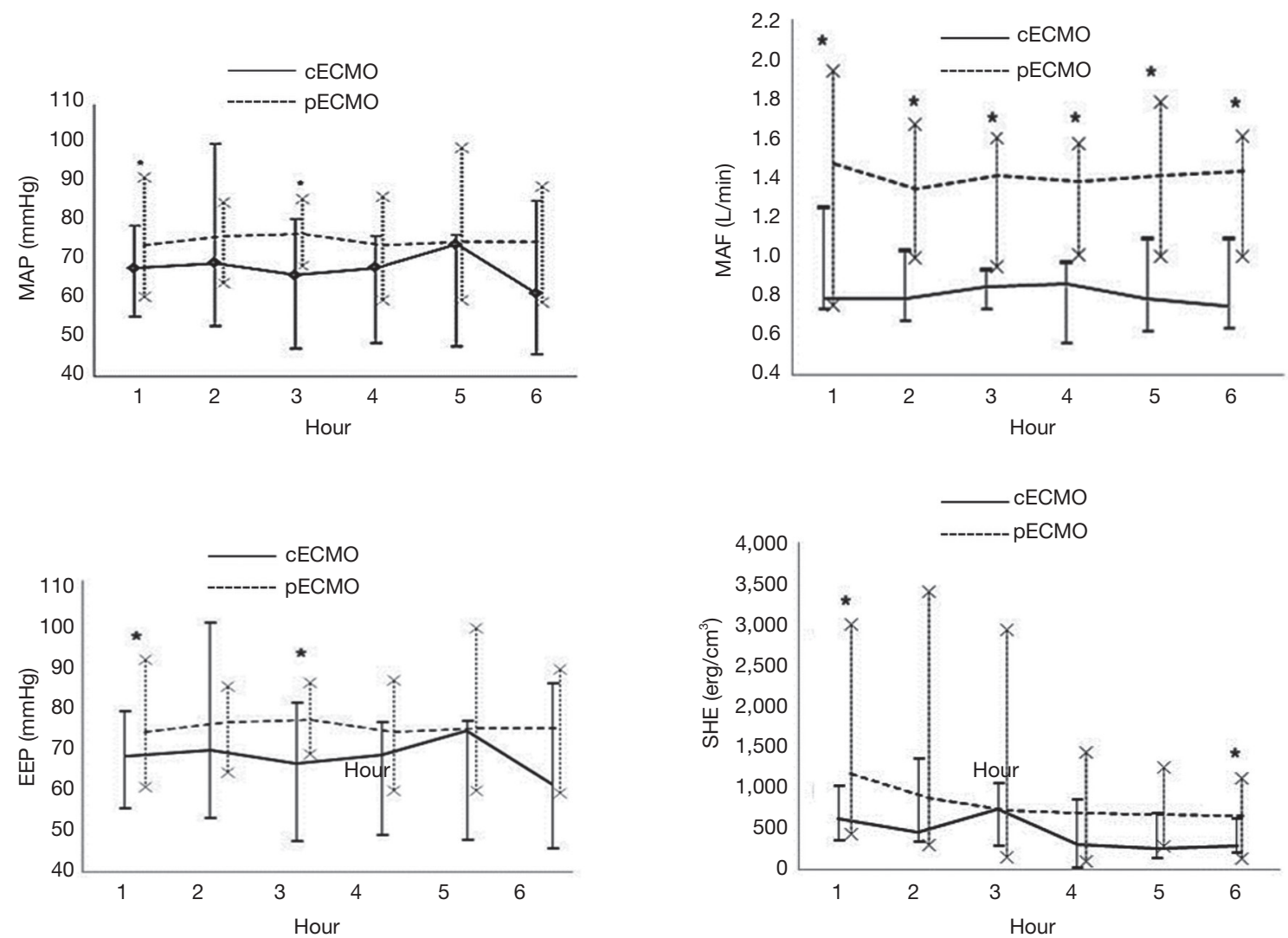

Figure 1 Comparison of hemodynamic parameters between cECMO and pECMO. Each line graph represents the median level of each hemodynamic parameter with minimal and maximal data. Between the two groups at each hour, ${ }^{*} \mathrm{P}<0.05$. cECMO, central ECMO group; pECMO, peripheral ECMO group; MAP, mean arterial pressure; MAF, mean arterial flow; EEP, energy equivalent pressure; SHE, surplus hemodynamic energy.

Kaufmann $e t$ al. measured the velocity of pulsatile flow from the left ventricle and non-pulsatile flow from a cannula in the ascending aorta transmitted through a cannula to the carotid artery using computational fluid dynamics (CFD) and particle image velocimetry in a silicone model. With both methods, they observed a decrease in flow velocity in the carotid artery and an increase flow velocity in the descending aorta upon insertion of the cannula. This phenomenon was attributed to skewing of the direction of the cannula jet toward the descending aorta (16). Gu et al. used a CFD simulation and reported a higher flow rate and lower wall shear stress in the carotid artery with pECMO than with cECMO due to greater flow in the direction of the carotid artery (17). In our study, we also confirmed greater blood flow to the brain in the pECMO group compared to the cECMO group, as measured in the brachiocephalic artery.

We chose S100B, GFAP, and NSE as biomarkers for brain damage. S100B and GFAP are proteins that are mostly present in high concentration in glial cells $(18,19)$. $\mathrm{S} 100 \mathrm{~B}$ protein, has been proposed as a well-established marker of brain damage and death, since elevated S100B concentrations in different biological fluids have been found in adults, infants, and fetuses at risk for brain damage (20). Bembea et al. measured plasma GFAP levels in patients on ECMO at 6, 12, and 24 hours after cannula insertion, and then every day until the end of ECMO; they reported a significantly higher level of GFAP in patients with newonset brain damage (9). Chen et al. measured S100B and NSE as markers of brain damage in patients on $\mathrm{V}-\mathrm{V}$ ECMO versus controls over 24 hours, but they did not find a statistically significant difference in S100B and NSE levels in the V-V ECMO group (3). There have also been studies looking at IL-6, a proinflammatory cytokine, in patients with brain damage. Ferreira et al. observed that patients with traumatic brain injury showed significantly higher 
Table 3 Hemodynamic data during 6-hour ECMO treatment

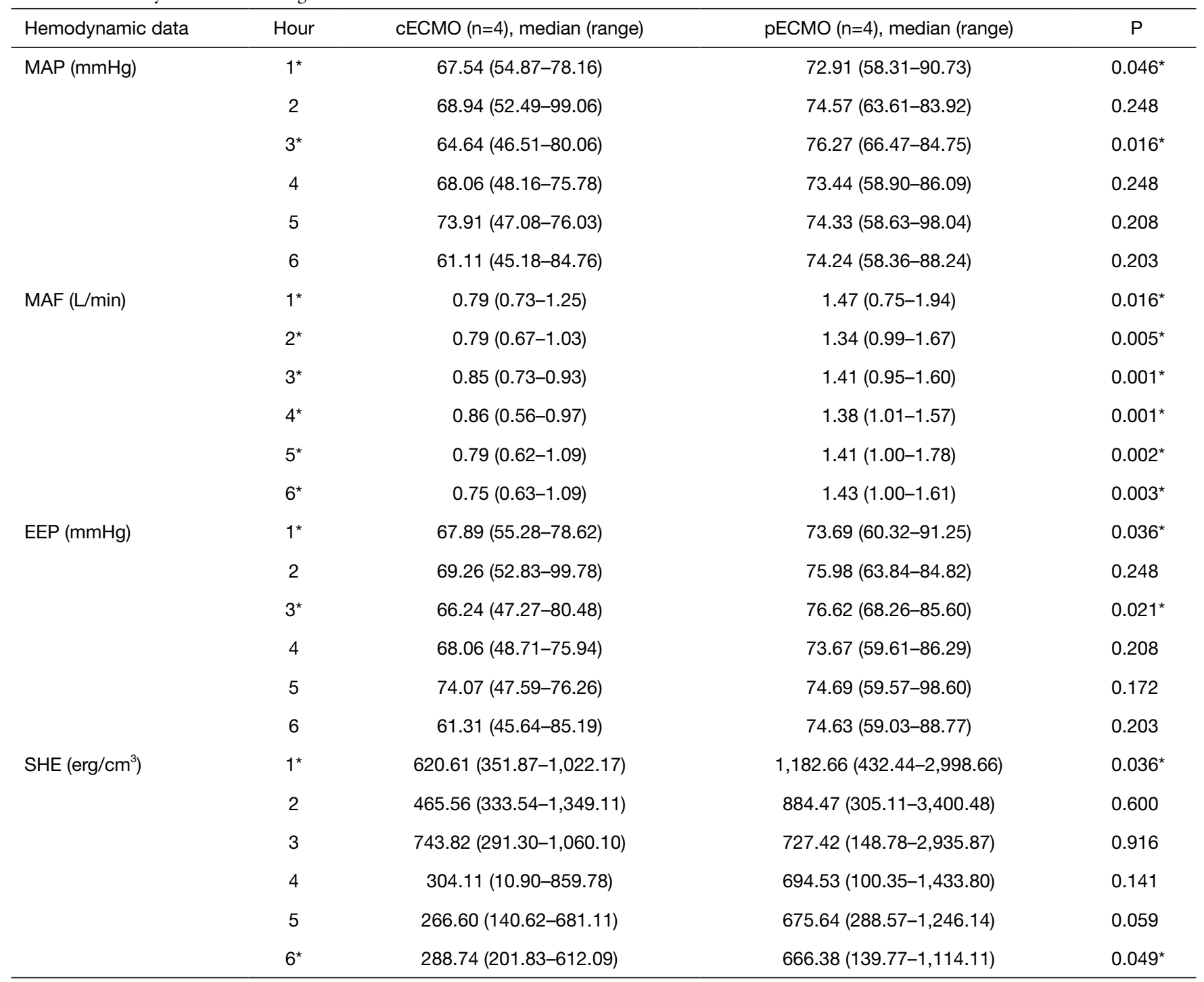

Between the two groups at each hour, ${ }^{\star} \mathrm{P}<0.05$. cECMO, central ECMO group; $\mathrm{PECMO}$, peripheral ECMO group; MAP, mean arterial pressure; MAF, mean arterial flow; EEP, energy equivalent pressure; SHE, surplus hemodynamic energy.

IL-6 levels 1 day after admission to the intensive care unit compared to healthy subjects (21). Chen $e$ al. also reported a statistically significant increase in plasma IL-6 levels 2 hours after initiation of V-V ECMO (3).

We speculated that V-A ECMO would cause more significant brain damage than V-V ECMO due to the loss of autoregulation, as the brain would then be supplied directly by non-pulsatile flow. The results of this study did not, however, show a significant increase in the plasma levels of S100B, GFAP, NSE, or IL-6 over time on V-A ECMO or a significant difference in any of these markers based on the site of cannula insertion. It is difficult to conclude no significant difference in extent of brain damage depending on the site of cannula insertion. Given that previous studies reported increasing markers of brain injury during 24 hours or longer $(3,8,9), 6$ hours of ECMO in this study were insufficient to observe a rise in brain damage markers. However, considering limitations of a longer-term experimental model such as hemorrhage, maintenance of blood pressure, and dilution of brain damage markers from continuous fluid resuscitation, it may be difficult to render an objective 

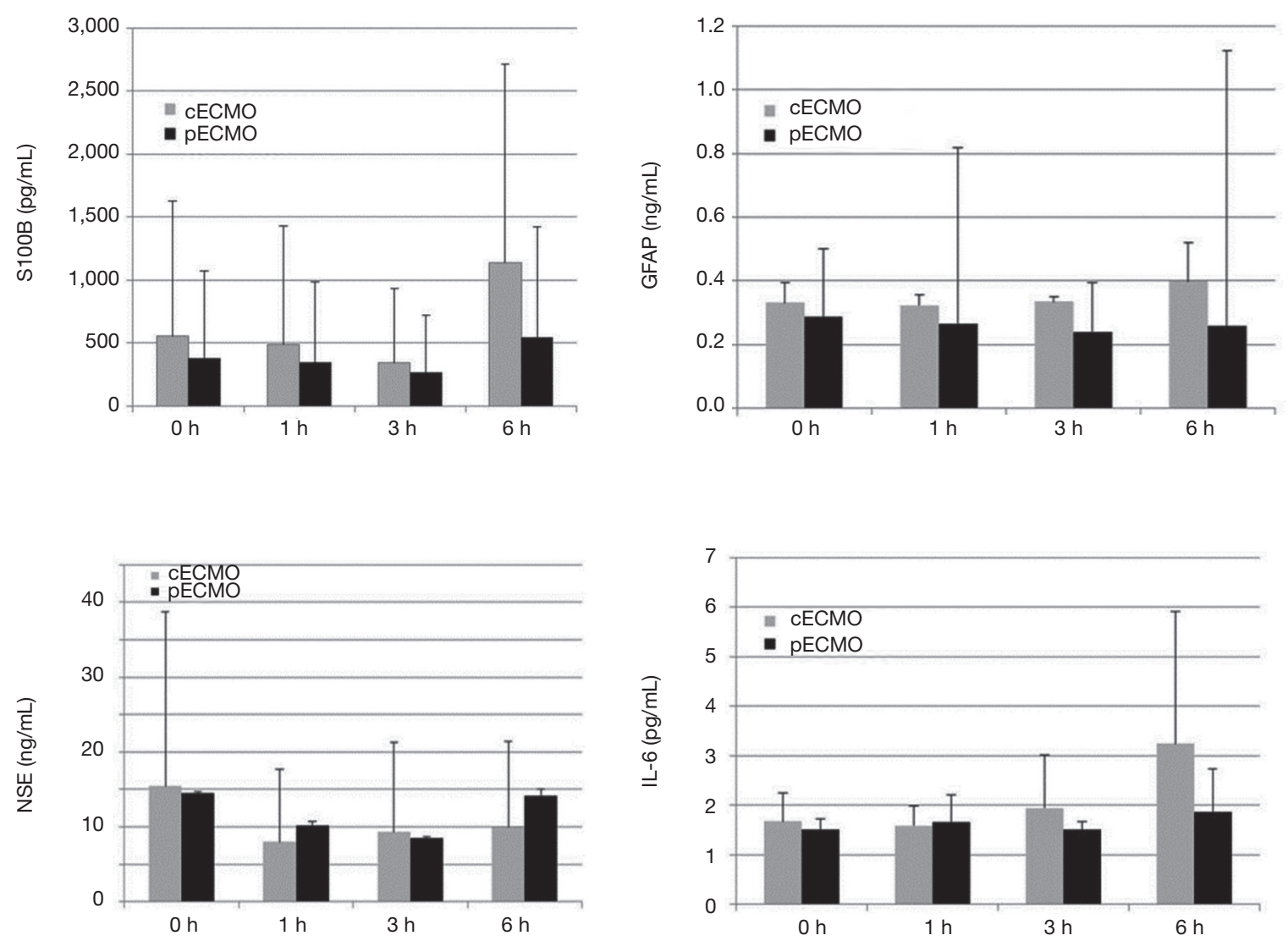

Figure 2 Serial plasma levels of biomarkers Serial plasma levels of S100B, GFAP, NSE and IL-6 were assessed at 0, 1, 3 and 6 hours during ECMO treatment. There was no statistical difference between cECMO and pECMO $(\mathrm{P}>0.05)$. cECMO, central ECMO group; pECMO, peripheral ECMO group; S100B, protein S100B; GFAP, glial fibrillary acidic protein; NSE, non-specific enolase; IL-6, interleukin-6.

relationship using animal models. Limitations of this study are as follows. First, analysis was non-parametric due to limited number of subjects. Although differences in levels of hemodynamic markers were statistically insignificant, most markers were higher in the peripheral ECMO group. It is possible this difference will reveal greater statistical significance with a greater number of subjects. Second, as mentioned above, it is difficult to predict the difference in extent of brain damage between two subject groups over a lengthier period of time because the rise in plasma markers of brain damage were not observed in this briefer 6-hour experimental model. Chen et al. failed to observe a rise in levels of brain damage markers after 24 hours of VV ECMO. However, they demonstrated that mRNA expression of IL-6, a proinflammatory cytokine, significantly upregulated in brain tissue (3). It is assumed that expression of such proinflammatory cytokines will subsequently cause damage to brain tissue. So, a significant rise in brain damage markers would be observed with a lengthier observation. Third, this study was conducted using healthy porcine subjects and cannot be directly applied to clinical settings, as ECMO is usually initiated on patients with left ventricular pathology or pulmonary compromise.

\section{Conclusions}

The hemodynamic data during ECMO management showed that $\mathrm{PECMO}$ achieved superior results compared with cECMO. However, 6-hour ECMO treatment did not increase brain injury markers with either $\mathrm{pECMO}$ or cECMO.

\section{Acknowledgements}

This study was supported by a Korea University Grant. 


\section{Footnote}

Conflicts of Interest: The authors have no conflicts of interest to declare.

Ethical Statement: This study was approved by the Guide for the Care and Use of Laboratory Animals issued by the Korea University School of Medicine (KUIACUC-2015-63).

\section{References}

1. Risnes I, Wagner K, Nome T, et al. Cerebral outcome in adult patients treated with extracorporeal membrane oxygenation. Ann Thorac Surg 2006;81:1401-6.

2. Marasco SF, Lukas G, McDonald M, et al. Review of ECMO (extra corporeal membrane oxygenation) support in critically ill adult patients. Heart Lung Circ 2008;17 Suppl 4:S41-7.

3. Chen $\mathrm{Q}, \mathrm{Yu} \mathrm{W}$, Shi J, et al. The effect of venovenous extra-corporeal membrane oxygenation (ECMO) therapy on immune inflammatory response of cerebral tissues in porcine model. J Cardiothorac Surg 2013;8:186.

4. Barrett CS, Bratton SL, Salvin JW, et al. Neurological injury after extracorporeal membrane oxygenation use to aid pediatric cardiopulmonary resuscitation. Pediatr Crit Care Med 2009;10:445-51.

5. Lidegran MK, Mosskin M, Ringertz HG, et al. Cranial CT for diagnosis of intracranial complications in adult and pediatric patients during ECMO: Clinical benefits in diagnosis and treatment. Acad Radiol 2007;14:62-71.

6. Campbell LR, Bunyapen C, Gangarosa ME, et al. Significance of seizures associated with extracorporeal membrane oxygenation. J Pediatr 1991;119:789-92.

7. Amigoni A, Pettenazzo A, Biban P, et al. Neurologic outcome in children after extracorporeal membrane oxygenation: prognostic value of diagnostic tests. Pediatr Neurol 2005;32:173-9.

8. Gazzolo D, Masetti P, Meli M, et al. Elevated S100B protein as an early indicator of intracranial haemorrhage in infants subjected to extracorporeal membrane oxygenation. Acta Paediatr 2002;91:218-21.

9. Bembea MM, Savage W, Strouse JJ, et al. Glial fibrillary acidic protein as a brain injury biomarker in children undergoing extracorporeal membrane oxygenation. Pediatr Crit Care Med 2011;12:572-9.

10. Undar A, Frazier OH, Fraser CD Jr. Defining pulsatile perfusion: quantification in terms of energy equivalent pressure. Artif Organs 1999;23:712-6.
11. Undar A, Rosenberg G, Myers JL. Major factors in the controversy of pulsatile versus nonpulsatile flow during acute and chronic cardiac support. ASAIO J 2005;51:173-5.

12. Shepard RB, Simpson DC, Sharp JF. Energy equivalent pressure. Arch Surg 1966;93:730-40.

13. Short BL, Walker LK, Bender KS, et al. Impairment of cerebral autoregulation during extracorporeal membrane oxygenation in newborn lambs. Pediatr Res 1993;33:289-94.

14. Hunter CJ, Blood AB, Bishai JM, et al. Cerebral blood flow and oxygenation during venoarterial and venovenous extracorporeal membrane oxygenation in the newborn lamb. Pediatr Crit Care Med 2004;5:475-81.

15. Liem KD, Hopman JC, Oeseburg B, et al. Cerebral oxygenation and hemodynamics during induction of extracorporeal membrane oxygenation as investigated by near infrared spectrophotometry. Pediatrics 1995;95:555-61.

16. Kaufmann TA, Neidlin $M$, Büsen $M$, et al. Implementation of intrinsic lumped parameter modeling into computational fluid dynamics studies of cardiopulmonary bypass. J Biomech 2014;47:729-35.

17. Gu K, Zhang Y, Gao B, et al. Hemodynamic Differences Between Central ECMO and Peripheral ECMO: A Primary CFD Study. Med Sci Monit 2016;22:717-26.

18. Fraser DD, Close TE, Rose KL, et al. Severe traumatic brain injury in children elevates glial fibrillary acidic protein in cerebrospinal fluid and serum. Pediatr Crit Care Med 2011;12:319-24.

19. Heizmann CW. Ca2+-binding S100 proteins in the central nervous system. Neurochem Res 1999;24:1097-100.

20. Varrica A, Satriano A, Frigiola A, et al. Circulating S100B and Adiponectin in Children Who Underwent Open Heart Surgery and Cardiopulmonary Bypass. Biomed Res Int 2015;2015:402642.

21. Ferreira LC, Regner A, Miotto KD, et al. Increased levels of interleukin-6, -8 and -10 are associated with fatal outcome following severe traumatic brain injury. Brain Inj 2014;28:1311-6.

Cite this article as: Jeong SC, Kim HJ, Shin YS, Han JW, Lim JY, Son HS. Influence of cannula positioning on brain injury during extracorporeal membrane oxygenation. J Thorac Dis 2018;10(11):6184-6191. doi: 10.21037/jtd.2018.10.74 